Supporting Information (SI)

\title{
Effects of Plasmonic Nanoparticle Incorporation on Electrodynamics and Photovoltaic Performance of Dye Sensitized Solar Cells
}

Julio Villanueva-Cab, Jose Luis Montaño-Priede, Umapada Pal

Instituto de Física, Benemérita Universidad Autónoma de Puebla, Apdo. Postal J-

48, Puebla, Pue.72570, México. E-mail: juliovc@ifuap.buap.mx

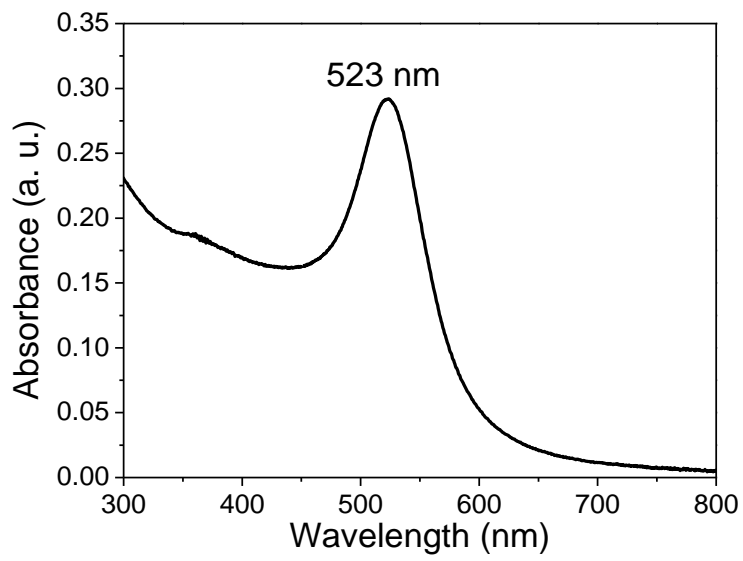

Figure S1. Absorption spectrum of the as-synthetized gold colloid. 

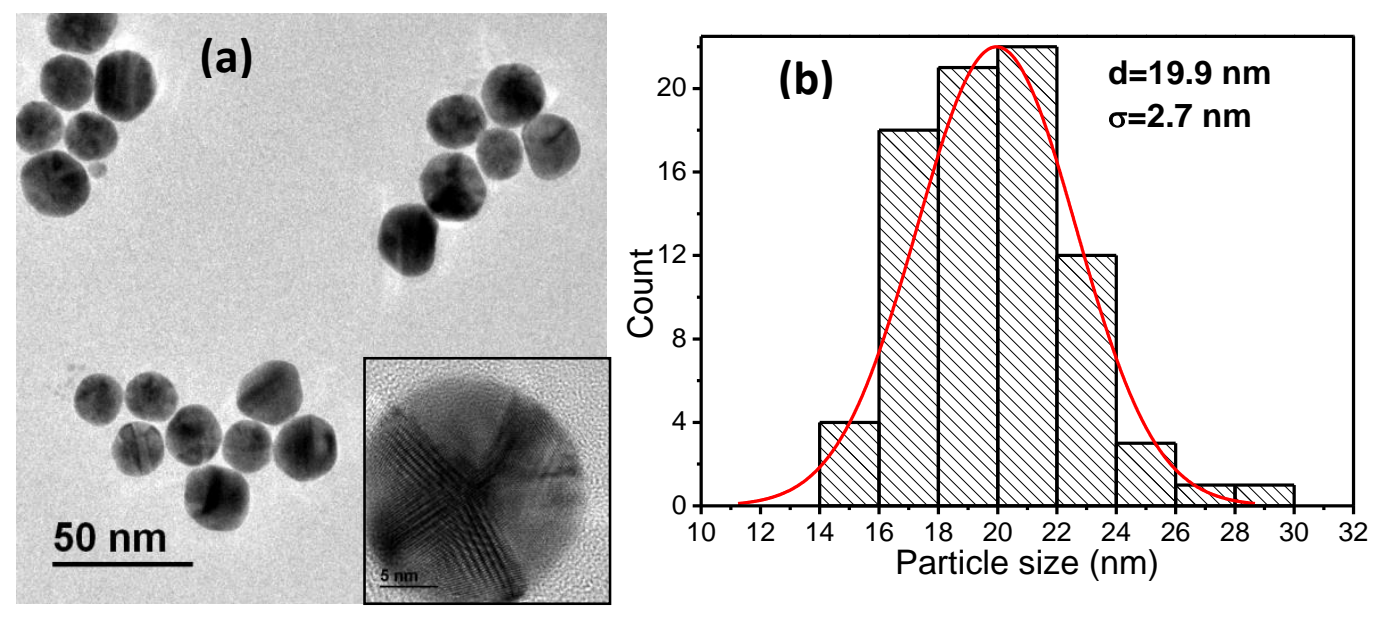

Figure S2. (a) Typical TEM micrograph of the gold nanoparticles. Inset shows the HRTEM micrograph of a representative Au nanoparticle, revealing its crystallinity and structural defects. (b) The size distribution histogram of Au nanoparticles and its Gaussian fit, revealing $19.9 \mathrm{~nm}$ average size and $13 \%$ of size dispersion.

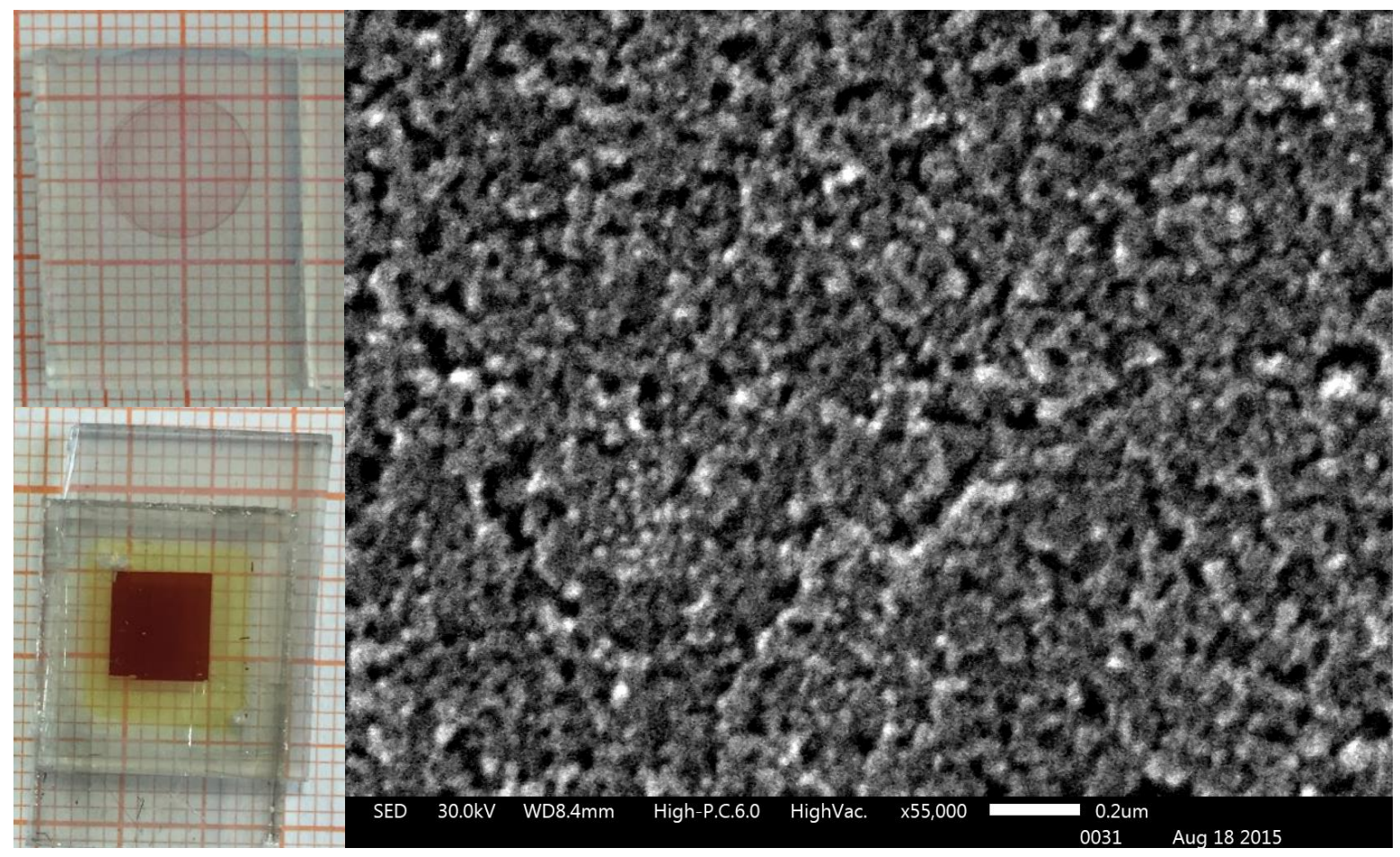

Figure S3. SEM image of the $\mathrm{TiO}_{2}$ film with $5 \mathrm{Au}$ NP treatments after firing at $350^{\circ} \mathrm{C}$. 


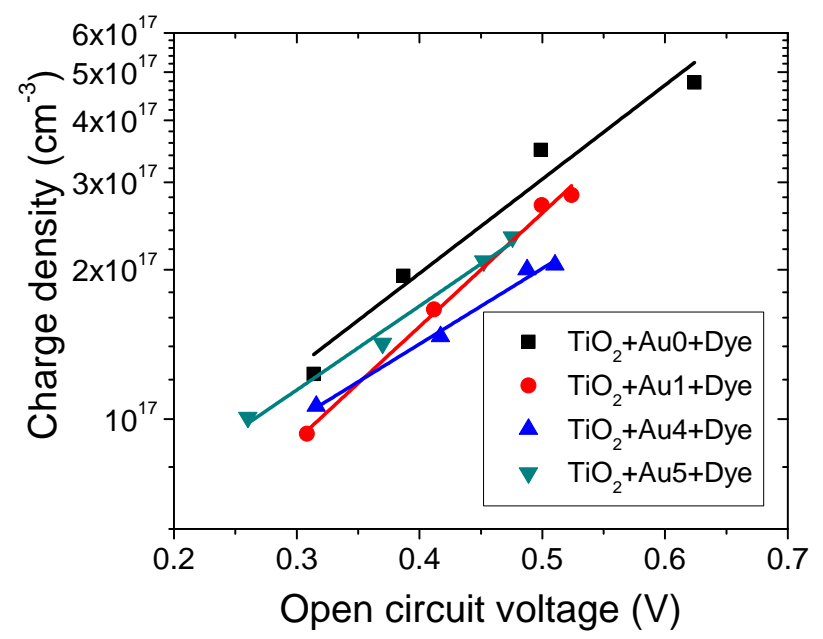

Figure S4. Electron charge density vs open circuit voltage plots as a function of light intensity for samples with different Au NP treatments.

The trap distribution parameter $\alpha^{\prime}$ was obtained by fitting the slope of the open circuit voltage $\left(V_{O C}\right)$ vs electron charge density $(n)$ plots using the relation: ${ }^{1}$

$n=n_{0} \exp \left[\alpha^{\prime} e V /\left(k_{\beta} T\right)\right]$

where $k_{\beta}$ is the Boltzmann constant, $e$ is the absolute value of electron charge, $T$ is the cell temperature $\left(25^{\circ} \mathrm{C}\right)$, and $V$ is the cell voltage. The electron charge density in dark $n_{0}$ is obtained by extrapolation of charge density to $V_{\mathrm{OC}}=0$. (dark condition). 


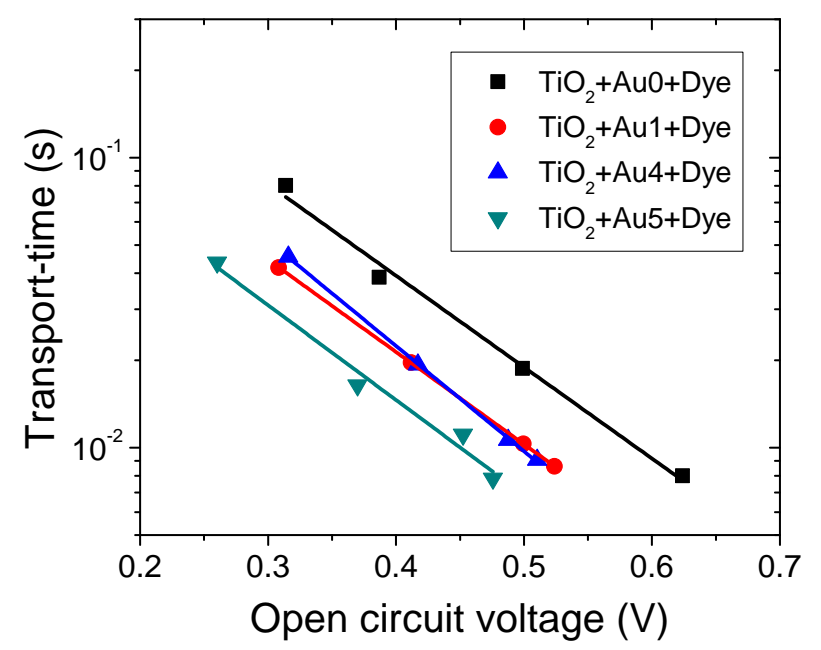

Figure S5. Electron transport-time vs open circuit voltage plots as a function of light intensity for samples with different Au NP treatments.

The non-ideality factor of free electron parameter ${ }^{2,3} b$ was obtained by fitting the slope of the electron transport-time $\tau_{\mathrm{tr}}$ (as obtained from SLIT of photovoltage measurements) vs open circuit voltage plot using the relation: ${ }^{1}$

$$
\tau_{t r}=\tau_{t r, 0} \exp \left[-\frac{(1-\alpha \prime b) e V}{\alpha \prime b\left(k_{\beta} T\right)}\right]
$$

and the previously determined $\alpha^{\prime}$ (Fig. S4). 


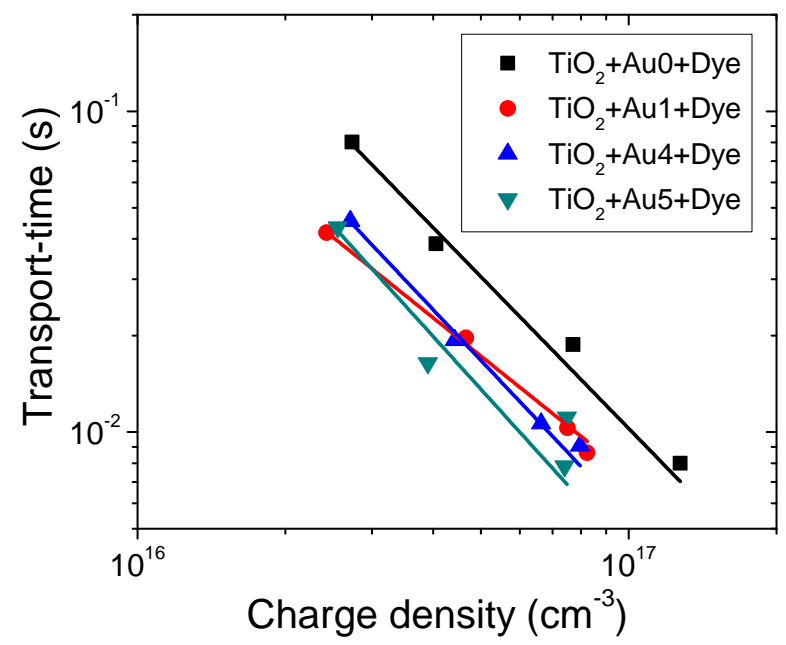

Figure S6. Electron transport-time vs charge density plots as a function of light intensity for samples with different Au NP treatments.

The transport-time in dark $\tau_{\mathrm{tr}, 0}$ was obtained by fitting the electron transport-time $\tau_{\mathrm{tr}}$ vs charge density plots using the relation: ${ }^{1}$

$$
\tau_{t r}=\tau_{t r, 0}\left[\frac{n}{n_{0}}\right]^{-\frac{1-\alpha \prime b}{\alpha \prime b}}
$$

and the previously estimated $\alpha$, and $b$ values (Figs. S4 \& S5) and extrapolating the fitted curves to $n=n_{0}$ (dark conditions) for each DSSCs with different Au NP treatments obtained from Figure S4. The diffusion coefficient in dark $D_{0}$ was obtained utilizing the relation: ${ }^{4}$

$$
D_{0}=\frac{d^{2}}{2.77 \tau_{t r, 0}}
$$




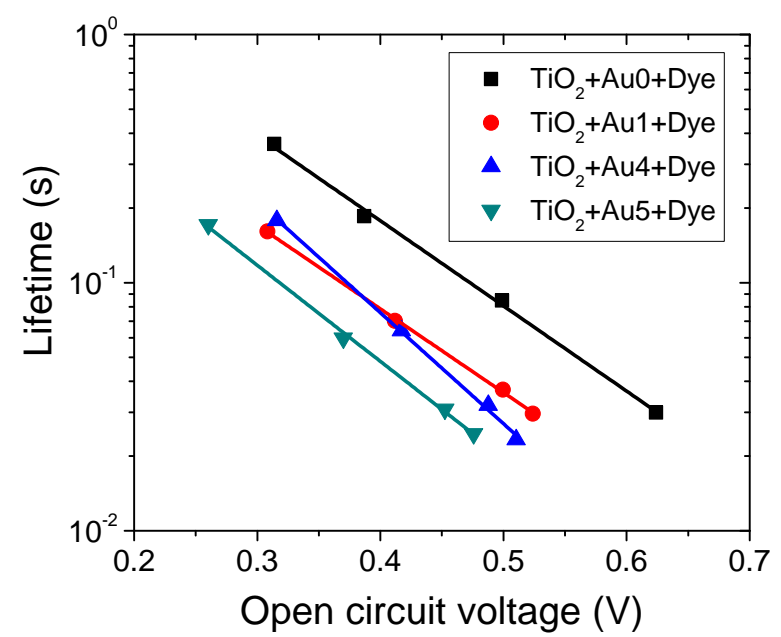

Figure S7. Electron lifetime vs open circuit voltage plots as a function of light intensity for samples with different Au NP treatments.

The nonlinear recombination parameter $\beta$ was obtained by fitting the slope of the electron lifetime $\tau_{\mathrm{r}}$ vs open circuit voltage plots using the relations: ${ }^{1}$

$$
\tau_{r}=\tau_{r, 0} \exp \left[-\frac{(\beta-\alpha \prime b) e V}{\alpha \prime b\left(k_{\beta} T\right)}\right]
$$

and the previously estimated $\alpha^{\prime}$ and $b$ values. The electron lifetime in dark $\tau_{r, 0}$ was obtained by extrapolating the fitting curves to $V_{\mathrm{OC}}=0$ (dark conditions) for each DSSCs with different Au NP treatments.

The as-obtained parameters are summarized on Table S1. These parameters were used to calculated the J-V curves with the aid of total electron density model (eq. 2 of the main text), assuming a total series resistance $R_{S}$ (Used to fit the experimental maximum power generated by the DSSCs). Figure S8 compares the calculated J-V curves with the corresponding experimental ones measured for DSSCs with different Au NP treatments. In Equation 2 of the main text the trap distribution parameter is defined as $\alpha=\alpha^{\prime} b$. 
Table S1. Fitting parameters obtained from SLIT of photovoltage and photocurrent measurements for the different Au treated DSSCs.

\begin{tabular}{lcccc}
\hline Parameter & Au0 & Au1 & Au4 & Au5 \\
\hline $\boldsymbol{\alpha}^{\mathbf{1}}$ & 0.114 & 0.137 & 0.091 & 0.101 \\
$\boldsymbol{\beta}$ & 1.0 & 1.0 & 1.0 & 1.0 \\
$\mathbf{b}$ & 3.308 & 3.053 & 3.242 & 3.367 \\
$\mathbf{n}_{\mathbf{0}}\left(\mathbf{m}^{-3}\right)$ & $2.83 \times 10^{16}$ & $1.84 \times 10^{16}$ & $2.19 \times 10^{16}$ & $3.08 \times 10^{16}$ \\
$\mathbf{D}_{\mathbf{0}}\left(\mathbf{m}^{\mathbf{2}} \mathbf{s}^{-1}\right)$ & $5.18 \times 10^{-11}$ & $6.00 \times 10^{-11}$ & $6.60 \times 10^{-11}$ & $1.25 \times 10^{-10}$ \\
$\mathbf{k}_{\mathbf{0}}\left(\mathbf{s}^{-1}\right) \mathbf{f i t}$ & 0.818 & 0.650 & 1.523 & 0.960 \\
$\mathbf{\eta}_{\text {inj }} \mathbf{f i t}$ & 0.596 & 0.498 & 0.472 & 0.426 \\
$\mathbf{d}(\boldsymbol{\mu m})$ & 3.6 & 3.6 & 3.6 & 3.6 \\
$\mathbf{R}_{\mathbf{S}}(\boldsymbol{\Omega})$ & 17.84 & 20.25 & 19.57 & 14.64 \\
\hline
\end{tabular}

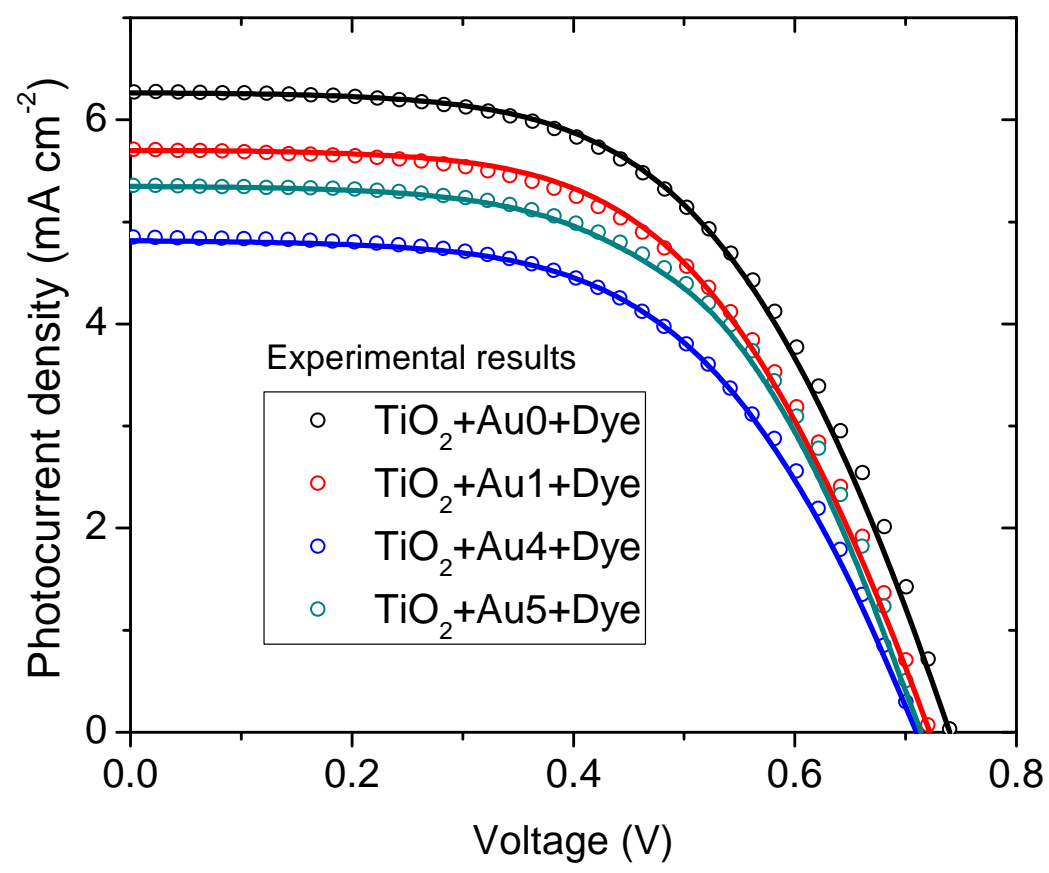

Figure S8. Comparison between the measured (symbols) and theoretical (continuous) current vs voltage curves for the DSSCs prepared with different Au NP treatments. 


\section{References}

1. Anta, J. A. et al. A Continuity Equation for the Simulation of the Current-Voltage Curve and the Time-Dependent Properties of Dye-Sensitized Solar Cells. Phys. Chem. Chem. Phys. 2012, 14, 10285-10299.

2. Jennings, J. R.; Ghicov, A.; Peter, L. M.; Schmuki, P.; Walker, A. B. Dye-Sensitized Solar Cells Based on Oriented $\mathrm{TiO}_{2}$ Nanotube Arrays: Transport, Trapping, and Transfer of Electrons. J. Am. Chem. Soc. 2008, 130, 13364-13372.

3. Barnes, P. R. F.; Anderson, A. Y.; Durrant, J. R.; O'Regan, B. C. Simulation and Measurement of Complete Dye Sensitised Solar Cells: Including the Influence of Trapping, Electrolyte, Oxidised Dyes and Light Intensity on Steady State and Transient Device Behaviour. Phys. Chem. Chem. Phys. 2011, 13, 5798-5816.

4. Nakade, S.; Kanzaki, T.; Wada, Y.; Yanagida, S. Stepped Light-Induced Transient Measurements of Photocurrent and Voltage in Dye-Sensitized Solar Cells: Application for Highly Viscous Electrolyte Systems. Langmuir 2005, 21, 10803-10807. 